\title{
Prediction of Compressive Strength of PFA- Cement Concrete
}

\author{
${ }^{1}$ L. Anyaogu, ${ }^{2}$ C. Chijioke and ${ }^{3}$ Okoye, $\mathrm{P}$ \\ ${ }^{I}$ Department of Civil Engineering, Federal University of Technology, Owerri, Nigeria \\ ${ }^{2}$ Department of Civil Engineering Federal Polytechnic, Nekede, Owerri, Nigeria \\ ${ }^{3}$ Department of Mechanical Engineering Federal Polytechnic Nekede Owerri Nigeria
}

\begin{abstract}
This work aims at prediction of compressive strength of Pulverised Fuel ash (PFA) - cement concrete based on Osadebe's five component second-degree polynomial. The model was used to optimize the compressive strength of concrete made from, cement, Pulverised Fuel Ash, sand, granites and water. A total of ninety (90) cubes were cast, comprising three cubes for each mix ratio and a total of thirty (30) mix ratios. The first fifteen (15) were used to determine the coefficients of the model, while the other fifteen were used to validate the model. The mathematical model compared favourably with the experimental data and the predictions from the model were tested with the statistical fischer test and found to be adequate at 95\% confidence level. With the model developed in this work, any desired compressive strength Pulverised Fuel Ashcement concrete can be predicted from known mix proportions and vice versa.

Keywords: Pulverised Fuel Ash; model; optimization; compressive strength; cement; concrete
\end{abstract}

\section{Introduction}

Construction industries in Nigeria rely heavily on cement for its operation on development of shelter and other infrastructural facilities. The provision of low cost but durable materials is almost universally recognized as one of the set-backs to improve housing conditions in Nigeria and other developing countries. Among the building materials in use today, the Ordinary Portland Cement is a vital element in all types of construction. Cement is very expensive. This has drastically reduced the construction of housing. Consequently, it has become absolutely difficult for majority of Nigeria citizens to own houses.

According to Neville, et al (1990), concrete is a product of water, cement and aggregate, when sufficiently hardened, is used in carrying various loads .However, in Nigeria due to the rapid rising of the cost of ordinary Portland cement (which is an important ingredient in concrete) there is need to develop cheap and replaceable substitute for cement. Consequently, many researches are being carried out on cheap and replaceable or complimentary substitutes for ordinary Portland cement. These researches are also aimed at putting into effective use industrial waste products.

The properties of concrete are controlled by the relative quantities of cement, aggregates and water mixed together both in plastic and hardened states. Also these properties can be improved by the addition of either a chemical admixture or supplementary cementitious material, which will make the number of components of concrete five (that is in addition to water, cement, coarse aggregate and fine aggregate). For this research work the components of concrete are cement, Pulverised Fuel Ash (PFA), fine aggregate, coarse aggregate and water, in this work, a mathematical model for the optimization of Compressive Strength of concrete based on Osadebe's second-degree polynomial is developed with different percentages of PFA as partial replacement of cement. This involves testing concrete from the different mix ratios where cement is partially replaced with PFA and developing a mathematical model that can be used to predict the compressive strength of the concrete given any mix ratio or predict mix ratios given a particular Compressive Strength of concrete.

\section{Osadebe's Concrete Optimization Theory}

Osadebe in his theory stated that concrete consists of four- component material. It is produced by mixing water, cement, fine aggregate (sand) and coarse aggregate. These ingredients are mixed in reasonable proportions to achieve desired strength of the concrete. In this paper, the fifth component, Pulverised Fuel Ash shall be added as one of the component materials of concrete.

Let us consider an arbitrary amount ' $\mathrm{S}$ ' of a given concrete mixture. Let the portion of the $\mathrm{i}^{\text {th }}$ component of the five constituent materials of the concrete be $S_{i}, i=1,2,3,4,5$. This was carried out with the principle of absolute mass.

$S_{1}+S_{2}+S_{3}+S_{4}+S_{5}=S$ 
Or

$\frac{S_{1}}{S}+\frac{S_{2}}{S}+\frac{S_{3}}{S}+\frac{S_{4}}{S}+\frac{S_{5}}{S}=1$

Where $\frac{S_{i}}{S}$ is the proportion of the $\mathrm{i}^{\text {th }}$ constituent component of the concrete mixture.

Let $\frac{s_{i}}{S}=Z_{i}, \quad i=1,2,3,4,5$

Substituting equation (3) into equation (2) yields:

$Z_{1}+Z_{2}+Z_{3}+Z_{4}+Z_{5}=1$

Experience has shown that the coefficient of regression when $\sum Z=1$ is mostly too large that the regression becomes too sensitive. As a result, when values of predictors outside the ones used in formulating the model are used to predict the response, the regression gives outrageous values. To correct this short coming a system of $Z$ that will make $\sum \mathrm{Z}=10$ will be adopted for convenience.

Multiplying equation (4) by 10 gives equation (4b)

$10 Z_{1}+10 Z_{2}+10 Z_{3}+10 Z_{4}+10 Z_{5}=10$

Let $10 Z_{\mathrm{i}}=\mathrm{Z}_{\mathrm{i}}$

Thus, $Z_{1}+Z_{2}+Z_{3}+Z_{4}+Z_{5}=10$

Where $Z_{1}, Z_{2}, Z_{3}, Z_{4}$ and $Z_{5}$ are proportions of water, cement, Pulverised Fuel Ash, sand, and coarse aggregate respectively. In general, for any given concrete mixture, exists a vector

$\mathrm{Z}\left(Z_{1}, Z_{2}, Z_{3}, Z_{4}\right)$. In this paper where five component materials are considered, the vector is transformed to $\mathrm{Z}$ $\left(Z_{1}, Z_{2}, Z_{3}, Z_{4}, Z_{5}\right)$ whose elements satisfy equation (4). Also, for each value of $Z_{i}$, the following inequality holds:

$$
Z_{i}>0
$$

It is important to note that the proportion of relative constituent ingredient of concrete govern the strength of the concrete at its hardened state. Thus, the compressive strength, $\mathrm{Y}$, of concrete can be expressed mathematically using equation (6) as:

$\mathrm{Y}=f\left(Z_{1}, Z_{2}, Z_{3}, Z_{4}, Z_{5}\right)$

Where $f\left(Z_{1}, Z_{2}, Z_{3} Z_{4}, Z_{5}\right)$ is a multi-variate response function whose variables $Z_{i}$ are subject to the constraints as defined in equations (4) and (5).

\section{Osadebe's regression equation}

This model assumed that the response function is continuous and differentiable with respect to its variables, $Z_{i}$, hence, it can be expanded using Taylor's series in the neighbourhood of a chosen point $Z^{(0)}=Z_{1}^{(0)}+Z_{2}^{(0)}+$ $Z_{3}^{(0)}+Z_{4}^{(0)}+Z_{5}^{(0)}$ as follows:

$f(Z)=\sum f^{m}\left(Z^{(0)}\right)+\frac{\left(Z_{i}-Z^{(0)}\right)}{m !}$

for $0 \leq m \leq \infty$

Where $\mathrm{m}$ is the degree of polynomial of the response function and $f(Z)$ is the response function. Expanding equation (7) to the second order yields: 


$$
\begin{aligned}
f(Z)=f\left(Z^{(0)}\right)+ & \sum_{i=1}^{5} \frac{\partial f\left(Z^{(0)}\right)}{\partial Z_{i}}\left(Z_{i}-Z_{i}^{(0)}\right)+\frac{1}{2} ! \sum_{i=1}^{4} \sum_{j=1}^{5} \frac{\partial^{2} f\left(Z^{(0)}\right)}{\partial Z_{i} \partial Z_{j}}\left(Z_{i}-Z_{i}^{(0)}\right)\left(Z_{j}-Z_{j}^{(0)}\right) \\
& +\frac{1}{2} ! \sum_{i=1}^{5} \frac{\partial^{2} f\left(Z^{(0)}\right)}{\partial Z_{i}^{2}}\left(Z_{i}-Z_{i}^{(0)}\right)^{2}+\cdots
\end{aligned}
$$

The point $Z^{(0)}$ will be chosen as the origin for convenience sake without loss of generality of the formulation. The predictor $Z_{i}$ is not the actual portion of the mixture component, rather. It is the ratio of the actual portions to the quantity of concrete. For convenience sake, let $Z_{i}$ be called the term of "fractional portion". The actual portions of the mixture components are $\mathrm{S}_{\mathrm{i}}$.

Consequently, the origin, $Z^{(0)}=0$.this implies that:

$\mathrm{Z}_{1}^{(0)}=0, \mathrm{Z}_{2}^{(0)}=0, \mathrm{Z}_{3}^{(0)}=0, \mathrm{Z}_{4}^{(0)}=0, \mathrm{Z}_{5}^{(0)}=0$

Let $b_{0}=f(0), b_{i}=\frac{\partial f(0)}{\partial Z_{i}}, b_{i j}=\frac{\partial^{2} f(0)}{\partial Z_{i} \partial Z_{j}}$ and $b_{i i}=\frac{\partial^{2} f(0)}{\partial Z_{i}^{2}}$

Equation (8) can then be written as follows:

$f(0)=b_{0}+\sum_{i=1}^{5} b_{i} Z_{i}+\sum_{i=1}^{4} \sum_{j=1}^{5} b_{i j} Z_{i} Z_{j}+\sum_{i=1}^{5} b_{i i} Z_{i}^{2}+\cdots$

Multiplying equation (4) by $b_{0}$, gives the following expression:

$b_{0}=b_{0} Z_{1}+b_{0} Z_{2}+b_{0} Z_{3}+b_{0} Z_{4}+b_{0} Z_{5}$

Similarly, multiplying equation (4) by $Z_{i}$ will give the following expression:

$Z_{1}=Z_{1}^{2}+Z_{1} Z_{2}+Z_{1} Z_{3}+Z_{1} Z_{4}+Z_{1} Z_{5}$

$Z_{2}=Z_{1} Z_{2}+Z_{2}^{2}+Z_{2} Z_{3}+Z_{2} Z_{4}+Z_{2} Z_{5}$

$Z_{3}=Z_{1} Z_{3}+Z_{2} Z_{3}+Z_{3}^{3}+Z_{3} Z_{4}+Z_{3} Z_{5}$

$\mathrm{Z}_{4}=\mathrm{Z}_{1} \mathrm{Z}_{4}+\mathrm{Z}_{2} \mathrm{Z}_{4}+\mathrm{Z}_{4} \mathrm{Z}_{3}+\mathrm{Z}_{4}^{2}+\mathrm{Z}_{4} \mathrm{Z}_{5}$

$Z_{5}=Z_{1} Z_{5}+Z_{2} Z_{5}+Z_{3} Z_{5}+Z_{4} Z_{5}+Z_{5}^{2}$

Rearranging equations (12a) to (12e), the expression for $Z_{i}^{2}$ becomes;

$Z_{1}^{2}=Z_{1}-Z_{1} Z_{2}-Z_{1} Z_{3}-Z_{1} Z_{4}-Z_{1} Z_{5}$

$\mathrm{Z}_{2}^{2}=\mathrm{Z}_{2}-\mathrm{Z}_{1} \mathrm{Z}_{2}-\mathrm{Z}_{2} \mathrm{Z}_{3}-\mathrm{Z}_{2} \mathrm{Z}_{4}-\mathrm{Z}_{2} \mathrm{Z}_{5}$

$Z_{3}^{2}=Z_{3}-Z_{1} Z_{3}-Z_{2} Z_{3}-Z_{3} Z_{4}-Z_{3} Z_{5}$

$\mathrm{Z}_{4}^{2}=\mathrm{Z}_{4}-\mathrm{Z}_{1} \mathrm{Z}_{4}-\mathrm{Z}_{2} \mathrm{Z}_{4}-\mathrm{Z}_{3} \mathrm{Z}_{4}-\mathrm{Z}_{4} \mathrm{Z}_{5}$

Substituting equation (13a) to (13e) into equation (10) and setting $f(0)=Y$ will give in the expanded form below:

$Y=b_{0} Z_{1}+b_{0} Z_{2}+b_{0} Z_{3}+b_{0} Z_{4}+b_{0} Z_{5}+b_{1} Z_{1}+b_{2} Z_{2}+b_{3} Z_{3}+b_{4} Z_{4}+b_{5} Z_{5}+b_{12} Z_{1} Z_{2}+$ 


$$
\begin{aligned}
& b_{13} Z_{1} Z_{1} Z_{3}+b_{14} Z_{1} Z_{4}+b_{15} Z_{1} Z_{5}+b_{23} Z_{2} Z_{3}+b_{24} Z_{2} Z_{4}+b_{25} Z_{2} Z_{5}+b_{34} Z_{3} Z_{4}+ \\
& b_{35} Z_{3} Z_{5}+b_{45} Z_{4} Z_{5}+b_{11}\left(Z_{1}-Z_{1} Z_{2}-Z_{1} Z_{3}-Z_{1} Z_{4}-Z_{1} Z_{5}\right)+ \\
& b_{22}\left(Z_{2}-Z_{1} Z_{2}-Z_{2} Z_{3}-Z_{2} Z_{4}-Z_{2} Z_{5}\right)+b_{33}\left(Z_{3}-Z_{1} Z_{3}-Z_{2} Z_{3}-Z_{3} Z_{4}-Z_{3} Z_{5}\right)+ \\
& b_{44}\left(Z_{4}-Z_{1} Z_{4}-Z_{2} Z_{4}-Z_{3} Z_{4}-Z_{4} Z_{5}\right)-b_{55}\left(Z_{5}-Z_{1} Z_{5}-Z_{2} Z_{5}-Z_{3} Z_{5}-Z_{4} Z_{5}\right)
\end{aligned}
$$

Factorizing equation (14a) yields

$$
\begin{aligned}
Y=Z_{1}\left(b_{0}+b_{1}+\right. & \left.b_{11}\right)+Z_{2}\left(b_{0}+b_{2}+b_{22}\right)+Z_{3}\left(b_{0}+b_{3}+b_{33}\right)+Z_{4}\left(b_{0}+b_{4}+b_{44}\right)+Z_{5}\left(b_{0}+b_{5}+b_{55}\right) \\
& +Z_{1} Z_{2}\left(b_{12}-b_{11}-b_{22}\right)+Z_{1} Z_{3}\left(b_{13}-b_{11}-b_{33}\right)+Z_{1} Z_{4}\left(b_{14}-b_{11}-b_{44}\right) \\
& +Z_{1} Z_{5}\left(b_{15}-b_{11}-b_{55}\right)+Z_{2} Z_{3}\left(b_{23}-b_{22}-b_{33}\right)+Z_{2} Z_{4}\left(b_{24}-b_{22}-b_{44}\right) \\
& +Z_{2} Z_{5}\left(b_{25}-b_{22}-b_{55}\right)+Z_{3} Z_{4}\left(b_{34}-b_{33}-b_{44}\right)+Z_{3} Z_{5}\left(b_{35}-b_{33}-b_{55}\right) \\
& +Z_{4} Z_{5}\left(b_{45}-b_{44}-b_{55}\right)
\end{aligned}
$$

The summation of the constants is equal to a constant thus; let

$$
\propto_{i}=b_{0}+b_{i}+b_{i i} \text { and } \propto_{i j}=b_{i j}+b_{i i}+b_{j j}
$$

Equation (14b) becomes:

$$
\begin{aligned}
Y= & \propto_{1} Z_{1}+\propto_{2} Z_{2}+\propto_{3} Z_{3}+\propto_{4} Z_{4}+\propto_{5} Z_{5}+\propto_{12} Z_{1} Z_{2}+\propto_{13} Z_{1} Z_{3}+\propto_{14} Z_{1} Z_{4}+\propto_{15} Z_{1} Z_{5}+\propto_{23} Z_{2} Z_{3}+ \\
& \propto_{24} Z_{2} Z_{4}+\propto_{25} Z_{2} Z_{5}+\propto_{34} Z_{3} Z_{4}+\propto_{35} Z_{3} Z_{5}+\propto_{45} Z_{4} Z_{5}
\end{aligned}
$$

or

$$
Y=\sum_{i=1}^{5} \alpha_{i} Z_{i}+\sum_{1 \leq i \leq j}^{5} \alpha_{i j} Z_{i} Z_{j}
$$

Where, $\mathrm{Y}$ is the response function at any point of observation $Z_{i}$ and $Z_{j}$ are the predictors; $\propto_{i}$ and $\propto_{i j}$ are the coefficient of the regression equation.

\section{The Coefficient of the Regression Equation}

Let $n^{\text {th }}$ response (compressive strength for the serial number $n$ ) be $Y^{(n)}$ and the vector of the corresponding set of variables be (see Table 1):

$$
Z^{(n)}=\left(Z_{1}^{(n)}, Z_{2}^{(n)}, Z_{3}^{(n)}, Z_{4}^{(n)}, Z_{5}^{(n)}\right)
$$

Different points of observation will have different predictor at constant coefficient. At $\mathrm{n}^{\text {th }}$ observation point, the response function $\mathrm{Y}^{(\mathrm{n})}$ will correspond with the predictors $\mathrm{Z}_{\mathrm{i}}^{(\mathrm{n})}$.

Thus,

$$
Y^{(n)}=\sum_{i=1}^{5} \alpha_{i} Z_{i}^{(n)}+\sum_{1 \leq i \leq j}^{5} \alpha_{i j} Z_{i} Z_{j}^{(n)}
$$

Where $1 \leq \mathrm{i} \leq \mathrm{j} \leq 5$ and $\mathrm{n}=1,2,3, \ldots, 15$

Equation (16) can be written in a matrix form as,

$\left[\mathrm{Y}^{(\mathrm{n})}\right]=\left[\mathrm{Z}^{(\mathrm{n})}\right][\propto]$

Expanding equation (17) gives: 


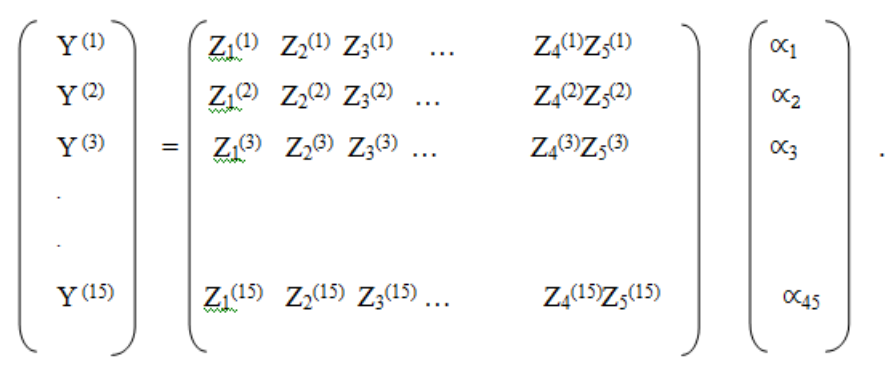

The actual mixture proportions $S_{i}{ }^{(n)}$ and the corresponding fractional portions, $Z_{i}^{(n)}$ is shown in table 1.

The values of the constant coefficient $\mathbf{a}$ in equation (17) are determined with the values of $Y^{(\mathrm{n})}$ and $Z^{(\mathrm{n})}$. Rearranging equation (17) gives

$[\propto]=\left[\mathrm{Z}^{(\mathrm{n})}\right]^{-1}\left[\mathrm{Y}^{(\mathrm{n})}\right]$

Expressing equation (19) in expanded form yields:

$$
\left(\begin{array}{c}
\propto_{1} \\
\propto_{2} \\
\propto_{3} \\
\propto_{45}
\end{array}\right)=\left(\begin{array}{lllll}
\mathrm{Z}_{1}{ }^{(1)} & \mathrm{Z}_{2}^{(1)} & \mathrm{Z}_{3}^{(1)} & \ldots & \mathrm{Z}_{4}^{(1)} \mathrm{Z}_{5}^{(1)} \\
\mathrm{Z}_{1}^{(2)} & \mathrm{Z}_{2}^{(2)} & \mathrm{Z}_{3}^{(2)} & \ldots & \mathrm{Z}_{4}^{(2)} \mathrm{Z}_{5}^{(2)} \\
\mathrm{Z}_{1}^{(3)} & \mathrm{Z}_{2}^{(3)} & \mathrm{Z}_{3}^{(3)} & \ldots & \mathrm{Z}_{4}^{(3)} \mathrm{Z}_{5}^{(3)} \\
& & & & \\
\mathrm{Z}_{1}^{(15)} & \mathrm{Z}_{2}^{(15)} & \mathrm{Z}_{3}^{(15)} & \ldots & \mathrm{Z}_{4}^{(15)} \mathrm{Z}_{5}^{(15)}
\end{array}\right)^{-1}\left(\begin{array}{c}
\mathrm{Y}^{(1)} \\
\mathrm{Y}^{(2)} \\
\mathrm{Y}^{(3)} \\
\\
\mathrm{Y}^{(15)}
\end{array}\right)
$$

The values of $\propto_{1}$ to $\propto_{45}$ are obtained from equation (19) and substituted into equation (15a) to obtain the regression equation. The values of $\left.Z^{(n)}\right]$ matrix is shown in table 2 ; the values inverse of $\left[Z^{(n)}\right]$ matrix is presented in table 3 , while the values of $\left[\mathrm{Y}^{(\mathrm{n})}\right]$ matrix are obtained from the experimental investigation.

\section{Materials and Methods}

The materials used for the laboratory test included:

i. Water used for this work was obtained from the premises of Federal University of Technology, Owerri, Nigeria. The water was clean and good for drinking.

ii. Dangote cement, a brand of ordinary Portland cement that conforms to BS 12(1978)

iii. The fine aggregate, river sand used for this research work were obtained from a Otamiri river, near Federal University of Technology, Owerri

iv. The coarse aggregate, granite chippings used was quarried from crushed rock industries quarry, Ishiagu, along Enugu-Port Harcourt express way, Ebonyi state, Nigeria. The granite has a maximum size of $20 \mathrm{~mm}$. They were washed and sun-dried for seven days in the laboratory to ensure that they were free from excessive dust, and organic matter.

v. Pulverised Fuel Ash (PFA) is a pozzolanic material. Pulverised Fuel Ash used as a partial replacement of cement in various mix proportion was obtained from the thermal coal station at Oji River, Enugu state, Nigeria. It was grinded and sieved $212 \mu \mathrm{m}$ sieve to obtain finer particles capable of reacting with cement, fine aggregate, coarse aggregates and water to form Pulverised Fuel Ash-cement concrete

The mix ratios used for the simplex design points were obtained using pentahedron factor space for five component mixture.

\section{Compressive Strength Test}

Batching of the ingredients was done by mass. Cement/ Pulverised Fuel Ash were thoroughly mixed together with a mixture of sand and granite. The entire component was cast in concrete mould of size $150 \mathrm{x}$ 150x $150 \mathrm{~mm}$. The concrete cubes were cured in a curing tank for 28 days and were crushed using universal testing machines. Compressive strength of the cubes was calculated using equation 20:

$$
\text { Compressive strength }=\frac{\text { compressive load of cube at failure }(\mathrm{N})}{\text { cross sectional area of mould }\left(\mathrm{mm}^{2}\right)}
$$

The results of the compressive test for laboratory and the model are shown in Table 4 
Table 1: Selcted mix proportion and corresponding component fractional based on Osadebe's seconddegree polynomial For system of $\sum Z=10$

\begin{tabular}{|c|c|c|c|c|c|c|c|c|c|c|}
\hline \multirow{3}{*}{$\mathbf{S} / \mathbf{N}$} & \multicolumn{10}{|c|}{ TRIAL MIXES } \\
\hline & \multicolumn{5}{|c|}{ MIX PROPORTIONS } & \multicolumn{5}{|c|}{ COMPONENT'S FRACTION } \\
\hline & $\mathbf{S}_{1}$ & $\mathbf{S}_{2}$ & $\mathbf{S}_{\mathbf{3}}$ & $\mathbf{S}_{4}$ & $\mathbf{S}_{5}$ & $Z_{1}$ & $Z_{2}$ & $\mathbf{Z}_{3}$ & $\mathbf{Z}_{4}$ & $\mathbf{Z}_{5}$ \\
\hline 1 & 0.57 & 0.95 & 0.05 & 2 & 4.0 & 0.75 & 1.25 & 0.07 & 2.64 & 5.28 \\
\hline 2 & 0.5 & \begin{tabular}{|l|}
0.90 \\
\end{tabular} & 0.10 & 1.2 & 2.4 & 0.98 & 1.76 & 0.20 & 2.35 & 4.71 \\
\hline 3 & 0.55 & \begin{tabular}{|l|}
0.85 \\
\end{tabular} & 0.15 & 1.5 & 2.0 & 1.09 & 1.68 & 0.30 & 2.97 & 3.96 \\
\hline 4 & 0.6 & \begin{tabular}{|l|}
0.80 \\
\end{tabular} & 0.20 & 2.1 & 4.2 & 0.76 & 1.01 & 0.25 & 2.66 & 5.32 \\
\hline 5 & 0.6 & \begin{tabular}{|l|}
0.75 \\
\end{tabular} & 0.25 & 1.2 & 4.0 & 0.88 & 1.10 & 0.37 & 1.76 & 5.88 \\
\hline 12 & 0.535 & \begin{tabular}{|l|}
0.925 \\
\end{tabular} & 0.75 & 1.6 & 3.2 & 0.84 & 1.46 & 0.12 & 2.53 & 5.05 \\
\hline 13 & 0.56 & 0.90 & 0.10 & 1.75 & 3.0 & 0.89 & 1.43 & 0.16 & 2.77 & 4.75 \\
\hline 14 & 0.585 & \begin{tabular}{|l}
0.875 \\
\end{tabular} & 0.125 & 2.05 & 4.1 & 0.76 & 1.13 & 0.16 & 2.65 & 5.30 \\
\hline 15 & 0.585 & \begin{tabular}{|l|}
0.85 \\
\end{tabular} & 0.15 & 1.6 & 4.0 & 0.81 & 1.18 & 0.21 & 2.23 & 5.57 \\
\hline 23 & 0.525 & 0.875 & 0.125 & 1.35 & 2.2 & 1.03 & 1.72 & 0.25 & 2.66 & 4.33 \\
\hline 24 & 0.55 & \begin{tabular}{|l|}
0.85 \\
\end{tabular} & 0.15 & 1.65 & 3.3 & 0.85 & 1.31 & 0.23 & 2.54 & 5.08 \\
\hline 25 & 0.55 & \begin{tabular}{|l|}
0.825 \\
\end{tabular} & 0.175 & 1.2 & 3.2 & 0.92 & 1.39 & 0.29 & 2.02 & 5.38 \\
\hline 34 & 0.575 & 0.825 & 0.175 & 1.8 & 3.1 & 0.89 & 1.27 & 0.27 & 2.78 & 4.79 \\
\hline 35 & 0.575 & \begin{tabular}{|l|}
0.8 \\
\end{tabular} & 0.20 & 1.35 & 3.0 & 0.97 & 1.35 & 0.34 & 2.28 & 5.06 \\
\hline 45 & 0.6 & 0.775 & 0.225 & 1.65 & 4.1 & 0.82 & 1.05 & 0.31 & 2.24 & 5.58 \\
\hline \multicolumn{11}{|c|}{ CONTROL } \\
\hline $\mathrm{C}_{1}$ & 0.54 & \begin{tabular}{|l|}
0.9 \\
\end{tabular} & 0.1 & 1.567 & 2.80 & 0.914 & 1.524 & 0.169 & 2.653 & 4.740 \\
\hline $\mathrm{C}_{2}$ & 0.573 & \begin{tabular}{|l|l|}
0.867 \\
\end{tabular} & 0.133 & 1.866 & 3.40 & 2.838 & 1.265 & 0.194 & 2.728 & 3.971 \\
\hline $\mathrm{C}_{3}$ & 0.590 & 0.833 & 0.167 & 1.766 & 5.066 & 0.715 & 1.122 & 0.225 & 2.319 & 5.478 \\
\hline $\mathrm{C}_{4}$ & 0.555 & \begin{tabular}{|l|}
0.875 \\
\end{tabular} & 0.125 & 1.7 & 3.15 & 0.867 & 1.366 & 0.195 & 2.654 & 4.918 \\
\hline $\mathrm{C}_{5}$ & 0.580 & \begin{tabular}{|l}
0.8375 \\
\end{tabular} & 0.1635 & 1.7 & 3.55 & 0.849 & 1.226 & 0.239 & 2.489 & 5.198 \\
\hline $\mathrm{C}_{6}$ & 0.555 & 0.8625 & 0.1375 & 1.475 & 3.1 & 0.705 & 1.407 & 0.224 & 2.406 & 5.051 \\
\hline $\mathrm{C}_{7}$ & 0.546 & \begin{tabular}{|l|}
0.925 \\
\end{tabular} & 0.0875 & 1.675 & 3.1 & 0.861 & 1.443 & 0.138 & 2.649 & 4.703 \\
\hline $\mathrm{C}_{8}$ & 0.580 & 0.825 & 0.175 & 1.475 & 3.5 & 0.885 & 1.259 & 0.267 & 2.250 & 5.339 \\
\hline $\mathrm{C}_{9}$ & 0.585 & \begin{tabular}{|l|}
0.89 \\
\end{tabular} & 0.11 & 1.76 & 3.32 & 0.841 & 1.341 & 0.166 & 2.691 & 5.002 \\
\hline $\mathrm{C}_{10}$ & 0.569 & 0.85 & 0.15 & 1.6 & 3.32 & 0.810 & 1.311 & 0.231 & 2.468 & 5.120 \\
\hline $\mathrm{C}_{11}$ & 0.571 & \begin{tabular}{|l|}
0.855 \\
\end{tabular} & 0.145 & 1.68 & 3.48 & 0.848 & 1.270 & 0.215 & 2.496 & 5.170 \\
\hline $\mathrm{C}_{12}$ & 0.567 & \begin{tabular}{|l|}
0.835 \\
\end{tabular} & 0.165 & 1.61 & 3.34 & 0.870 & 1.281 & 0.253 & 2.470 & 5.125 \\
\hline $\mathrm{C}_{13}$ & 0.562 & 0.8675 & 0.1325 & 1.555 & 3.26 & 0.881 & 1.360 & 0.208 & 2.438 & 5.112 \\
\hline $\mathrm{C}_{14}$ & 0.562 & 0.855 & 0.145 & 1.625 & 3.42 & 0.855 & 1.293 & 0.2119 & 2.458 & 5.174 \\
\hline $\mathrm{C}_{15}$ & 0.582 & \begin{tabular}{|l}
0.8573 \\
\end{tabular} & 0.1425 & 1.74 & 3.96 & 0.725 & 1.178 & 0.176 & 2.389 & 5.438 \\
\hline
\end{tabular}

Table 2: $Z^{(n)}$ Matrix

\begin{tabular}{|l|l|l|l|l|l|l|l|l|l|l|l|l|l|l|}
\hline $\mathbf{Z}_{\mathbf{1}}$ & $\mathbf{Z}_{\mathbf{2}}$ & $\mathbf{Z}_{\mathbf{3}}$ & $\mathbf{Z}_{\mathbf{4}}$ & $\mathbf{Z}_{\mathbf{5}}$ & $\mathbf{Z}_{\mathbf{1}} \mathbf{Z}_{\mathbf{2}}$ & $\mathbf{Z}_{\mathbf{1}} \mathbf{Z}_{\mathbf{3}}$ & $\mathbf{Z}_{\mathbf{1}} \mathbf{Z}_{\mathbf{4}}$ & $\mathbf{Z}_{\mathbf{1}} \mathbf{Z}_{\mathbf{5}}$ & $\mathbf{Z}_{\mathbf{2}} \mathbf{Z}_{\mathbf{3}}$ & $\mathbf{Z}_{\mathbf{2}} \mathbf{Z}_{\mathbf{4}}$ & $\mathbf{Z}_{\mathbf{2}} \mathbf{Z}_{\mathbf{5}}$ & $\mathbf{Z}_{\mathbf{3}} \mathbf{Z}_{\mathbf{4}}$ & $\mathbf{Z}_{\mathbf{3}} \mathbf{Z}_{\mathbf{5}}$ & $\mathbf{Z}_{\mathbf{4}} \mathbf{Z}_{\mathbf{5}}$ \\
\hline 0.75 & 1.25 & 0.07 & 2.64 & 5.28 & 0.938 & 0.053 & 1.980 & 3.96 & 0.088 & 3.3 & 6.6 & 0.185 & 0.370 & 13.939 \\
\hline 0.98 & 1.76 & 0.20 & 2.35 & 4.71 & 1.725 & 0.196 & 2.303 & 4.616 & 0.352 & 4.136 & 8.290 & 0.47 & 0.942 & 11.069 \\
\hline 1.09 & 1.68 & 0.30 & 2.97 & 3.96 & 1.831 & 0.327 & 3.237 & 4.316 & 0.504 & 4.99 & 6.653 & 0.891 & 1.188 & 11.761 \\
\hline 0.76 & 1.01 & 0.25 & 2.66 & 5.32 & 0.768 & 0.19 & 2.002 & 4.043 & 0.253 & 2.687 & 5.373 & 0.665 & 1.33 & 14.151 \\
\hline 0.88 & 1.10 & 0.37 & 1.76 & 5.88 & 0.968 & 0.326 & 1.549 & 5.174 & 0.407 & 1.936 & 6.468 & 0.652 & 2.176 & 10.349 \\
\hline 0.84 & 1.46 & 0.12 & 2.53 & 5.05 & 1.226 & 0.101 & 2.125 & 4.242 & 0.175 & 3.694 & 7.373 & 0.304 & 0.606 & 12.777 \\
\hline 0.89 & 1.43 & 0.16 & 2.77 & 4.75 & 1.273 & 0.142 & 2.465 & 4.228 & 0.229 & 3.961 & 6.793 & 0.443 & 0.760 & 13.158 \\
\hline 0.76 & 1.13 & 0.16 & 2.65 & 5.30 & 0.859 & 0.122 & 2.014 & 4.028 & 0.021 & 2.995 & 5.989 & 0.424 & 0.848 & 14.045 \\
\hline 0.81 & 1.18 & 0.21 & 2.23 & 5.57 & 0.956 & 0.170 & 1.806 & 4.512 & 0.248 & 2.631 & 6.573 & 0.468 & 1.170 & 12.421 \\
\hline 1.03 & 1.72 & 0.25 & 2.66 & 4.33 & 1.772 & 0.258 & 2.740 & 4.460 & 0.430 & 4.575 & 7.448 & 0.665 & 1.083 & 11.518 \\
\hline 0.85 & 1.31 & 0.23 & 2.54 & 5.08 & 1.114 & 0.196 & 2.159 & 4.318 & 0.301 & 3.327 & 6.655 & 0.584 & 1.168 & 12.903 \\
\hline 0.92 & 1.39 & 0.29 & 2.02 & 5.38 & 1.279 & 0.267 & 1.858 & 4.950 & 0.403 & 2.808 & 7.478 & 0.586 & 1.560 & 10.868 \\
\hline
\end{tabular}


Prediction Of Compressive Strength Of Pfa-Cement Concrete

\begin{tabular}{|l|l|l|l|l|l|l|l|l|l|l|l|l|l|l|}
\hline 0.89 & 1.27 & 0.27 & 2.78 & 4.79 & 1.130 & 0.240 & 2.474 & 4.264 & 0.343 & 3.531 & 6.083 & 0.751 & 1.293 & 13.316 \\
\hline 0.97 & 1.35 & 0.34 & 2.28 & 5.06 & 1.310 & 0.330 & 2.212 & 4.108 & 0.459 & 3.078 & 6.831 & 0.775 & 1.720 & 11.537 \\
\hline 0.82 & 1.05 & 0.31 & 2.24 & 5.58 & 0.861 & 0.254 & 1.837 & 4.576 & 0.326 & 2.352 & 5.859 & 0.694 & 1.730 & 12.499 \\
\hline
\end{tabular}

Table 3: inverse of the $Z^{(n)}$ matrix

\begin{tabular}{|c|c|c|c|c|c|c|c|c|c|c|c|c|c|c|}
\hline$Z_{1}$ & $\mathbf{Z}_{2}$ & $\mathrm{Z}_{3}$ & $\mathrm{Z}_{4}$ & $Z_{5}$ & $\mathrm{Z}_{1} \mathrm{Z}_{2}$ & $\mathrm{Z}_{1} \mathrm{Z}_{3}$ & $\mathrm{Z}_{1} \mathrm{Z}_{4}$ & $\mathrm{Z}_{1} \mathrm{Z}_{5}$ & $\mathrm{Z}_{2} \mathrm{Z}_{3}$ & $\mathrm{Z}_{2} \mathrm{Z}_{4}$ & $\mathrm{Z}_{2} \mathrm{Z}_{5}$ & $Z_{3} Z_{4}$ & $\mathrm{Z}_{3} \mathrm{Z}_{5}$ & $\mathrm{Z}_{4} \mathrm{Z}_{5}$ \\
\hline-646.173 & -338.434 & 18.365 & 27.864 & 21.896 & 1177.784 & -374.486 & 931.8606 & -500.627 & 609.8273 & -1077.1 & 690.5306 & 194.6381 & -169.875 & 37.4698 \\
\hline-129.648 & 21.18276 & -25.2242 & -338.434 & -37.5063 & \begin{tabular}{|l|l}
134.9698 \\
\end{tabular} & -171.024 & \begin{tabular}{|l|l}
452.3441 \\
\end{tabular} & \begin{tabular}{|l}
-177.157 \\
\end{tabular} & \begin{tabular}{|l|l|}
42.70483 \\
\end{tabular} & .252 .712 & \begin{tabular}{|l|}
76.93981 \\
\end{tabular} & \begin{tabular}{|l|l}
249.7454 \\
\end{tabular} & -105.456 & 259.5039 \\
\hline 2413.256 & \begin{tabular}{|l|l|}
423.6017 \\
\end{tabular} & \begin{tabular}{|l|l}
-142.146 \\
\end{tabular} & 1389.092 & $\begin{array}{l}-8.13604 \\
\end{array}$ & -2510.62 & 1089.761 & -4040.68 & \begin{tabular}{|l|l|}
1019.163 \\
\end{tabular} & -97.585 & 1689.451 & \begin{tabular}{|l|}
-311.453 \\
\end{tabular} & \begin{tabular}{|l|}
-392.897 \\
\end{tabular} & -47.2449 & \\
\hline 9.979907 & \begin{tabular}{|l}
12.37629 \\
\end{tabular} & 1.105274 & 37.06226 & 20.04836 & -19.1537 & 8.127822 & -355.5584 & 28.19848 & -10.5298 & 20.23001 & -21.0455 & \begin{tabular}{|l|l}
-3.39347 \\
\end{tabular} & 8.456402 & \\
\hline-0.70243 & -1.21023 & 2.260212 & -1.23329 & -0.661 & 2.883123 & -2.44006 & \begin{tabular}{|l}
2.725709 \\
\end{tabular} & -1.794 & \begin{tabular}{|l|}
-0.44122 \\
\end{tabular} & -2.35277 & \begin{tabular}{|l|l}
3.356586 \\
\end{tabular} & \begin{tabular}{|l|}
-0.33916 \\
\end{tabular} & -3.15008 & 3.199157 \\
\hline 139.0292 & 101.2965 & 27.81165 & 166.3936 & 47.1372 & \begin{tabular}{|l|l} 
& -220.745 \\
\end{tabular} & 118.4014 & \begin{tabular}{|l|}
-299.209 \\
\end{tabular} & \begin{tabular}{|l|l}
139.1869 \\
\end{tabular} & -106.941 & 239.7851 & \begin{tabular}{|l|l}
-131.567 \\
\end{tabular} & -124.554 & \begin{tabular}{|l}
66.72179 \\
\end{tabular} & -162.907 \\
\hline-377.35 & 49.23741 & \begin{tabular}{|l|l}
64.51633 \\
\end{tabular} & -256.673 & 50.67247 & 343.061 & -195.925 & \begin{tabular}{|l|l}
690.3984 \\
\end{tabular} & \begin{tabular}{|l|l|}
-149.966 \\
\end{tabular} & \begin{tabular}{|l}
-112.639 \\
\end{tabular} & -165.384 & \begin{tabular}{|l|}
.79 .6911 \\
\end{tabular} & \begin{tabular}{|l|l}
51.33869 \\
\end{tabular} & 65.60519 & 52454 \\
\hline 49.55029 & 69.83678 & 19.51135 & -40.4485 & -1.95064 & -98.9843 & 26.11717 & -21.904 & -3.57826 & -69.4002 & 65.61689 & -26.0892 & \begin{tabular}{|l|l|}
4.513161 \\
\end{tabular} & -10.0775 & 37.19953 \\
\hline 85.16856 & \begin{tabular}{|l}
86.98385 \\
\end{tabular} & \begin{tabular}{|l}
7.106181 \\
\end{tabular} & 37.8435 & 34.42751 & \begin{tabular}{|l}
-154.081 \\
\end{tabular} & 47.67634 & \begin{tabular}{|l}
-132.674 \\
\end{tabular} & 81.67711 & \begin{tabular}{|l|}
-66.1678 \\
\end{tabular} & 138.788 & \begin{tabular}{|l|}
-100.151 \\
\end{tabular} & \begin{tabular}{|l|}
-19.1525 \\
\end{tabular} & 27.25763 & -74.7882 \\
\hline & -44.0953 & -2.21373 & -120.989 & -10.494 & 141.6209 & .71 .4494 & 287.8785 & .73 .5851 & 35.67465 & $\begin{array}{l}-111.037 \\
\end{array}$ & \begin{tabular}{|l}
50.14617 \\
\end{tabular} & 41.22743 & \begin{tabular}{|l}
-20.8249 \\
\end{tabular} & 52.50349 \\
\hline 1620087 & 617306 & 11345 & 44.80269 & 0237200 & -15.7313 & 178185 & \begin{tabular}{|l}
-62.5088 \\
\end{tabular} & \begin{tabular}{|l|l}
31.65995 \\
\end{tabular} & \begin{tabular}{|l|l}
3.707483 \\
\end{tabular} & 39.26193 & -20.9153 & \begin{tabular}{|l|}
-31.0027 \\
\end{tabular} & \begin{tabular}{|l}
7.11072 \\
\end{tabular} & -42.1414 \\
\hline 4.480164 & -8.14336 & 801717 & 26.28411 & -1.06366 & \begin{tabular}{|l|l}
1.400644 \\
\end{tabular} & 12.61005 & \begin{tabular}{|l}
-28.8556 \\
\end{tabular} & 4.92917 & \begin{tabular}{|l|}
-2.98015 \\
\end{tabular} & 12.14311 & \begin{tabular}{|l|}
5.002887 \\
\end{tabular} & \begin{tabular}{|l|}
-22.1311 \\
\end{tabular} & 6.388119 & -13.8778 \\
\hline-217.843 & -37.032 & 9.710062 & -89.2821 & \begin{tabular}{|l}
12.73257 \\
\end{tabular} & \begin{tabular}{|l|l|}
227.0139 \\
\end{tabular} & -98.6144 & 324.6998 & \begin{tabular}{|l}
.50 .5042 \\
\end{tabular} & \begin{tabular}{|l|l}
11.44769 \\
\end{tabular} & $\begin{array}{l}-134.587 \\
\end{array}$ & \begin{tabular}{|l|l|}
-1.63347 \\
\end{tabular} & \begin{tabular}{|l|}
27.82893 \\
\end{tabular} & 21.13685 & -5.13301 \\
\hline-258.563 & -54.4648 & \begin{tabular}{|l|l}
15.37428 \\
\end{tabular} & -149.431 & -7.14505 & \begin{tabular}{|l}
275.3811 \\
\end{tabular} & -110.394 & $\mid 432.9805$ & -126.299 & 15.0154 & -190.351 & \begin{tabular}{|l}
53.33176 \\
\end{tabular} & 37.376 & $\begin{array}{l}3.20157 \\
\end{array}$ & 70.33064 \\
\hline-1.65721 & -2.08884 & \begin{tabular}{|l|}
-0.63172 \\
\end{tabular} & -3.27577 & -2.66602 & 2.88902 & -0.57328 & \begin{tabular}{|l}
4.433649 \\
\end{tabular} & -3.78464 & 2.044158 & -2.56061 & 2.406579 & \begin{tabular}{|l|}
-0.94574 \\
\end{tabular} & 0.237125 & 6.173588 \\
\hline
\end{tabular}

Table 4: Compressive strength in $\mathbf{N} / \mathbf{m m}^{2}$ of $28^{\text {th }}$ day old concrete cubes

\begin{tabular}{|c|c|c|c|c|c|}
\hline $\begin{array}{l}\text { Point of } \\
\text { observation }\end{array}$ & $\begin{array}{l}\text { Compressive } \\
\text { strength of } \\
\text { Replication } 1 \\
\left(\mathrm{~N} / \mathrm{mm}^{2}\right)\end{array}$ & $\begin{array}{l}\text { Compressive } \\
\text { strength of } \\
\text { Replicate } 2 \\
\left(\mathrm{~N} / \mathrm{mm}^{2}\right)\end{array}$ & $\begin{array}{l}\text { Compressive } \\
\text { strength of } \\
\text { Replicate } 3 \\
\left(\mathrm{~N} / \mathrm{mm}^{2}\right)\end{array}$ & $\begin{array}{l}\text { Mean } \\
\text { compressive } \\
\text { strength }\left(\mathrm{N} / \mathrm{mm}^{2}\right)\end{array}$ & $\begin{array}{l}\text { Osadebe } \\
\text { model } \\
\text { compressive } \\
\text { strength } \\
\text { result } \\
\left(\mathrm{N} / \mathrm{mm}^{2}\right)\end{array}$ \\
\hline 1 & 42.66 & 42.66 & 42.22 & 42.51 & 42.51 \\
\hline 2 & 34.22 & 39.55 & 40.88 & 38.21 & 38.21 \\
\hline 3 & 31.55 & 34.46 & 34.22 & 33.41 & 33.41 \\
\hline 4 & 41.33 & 42.66 & 38.22 & 40.73 & 40.73 \\
\hline 5 & 21.33 & 24.44 & 20.00 & 21.92 & 21.92 \\
\hline 12 & 41.33 & 39.11 & 47.55 & 42.66 & 42.66 \\
\hline 13 & 40.88 & 40.00 & 40.77 & 40.55 & 40.55 \\
\hline 14 & 35.11 & 35.55 & 32.00 & 34.22 & 34.22 \\
\hline 15 & 31.11 & 28.44 & 28.88 & 29.47 & 29.47 \\
\hline 23 & 30.22 & 32.88 & 31.11 & 31.40 & 31.40 \\
\hline 24 & 33.77 & 34.22 & 33.77 & 33.92 & 33.92 \\
\hline 25 & 33.33 & 31.11 & 35.55 & 33.23 & 33.33 \\
\hline 34 & 26.66 & 29.33 & 29.77 & 28.58 & 28.58 \\
\hline 35 & 24.88 & 29.33 & 27.11 & 27.10 & 27.10 \\
\hline 45 & 17.77 & 18.66 & 18.66 & 18.36 & 18.36 \\
\hline $\mathrm{C}_{1}$ & 38.11 & 42.66 & 43.55 & 41.44 & 41.58 \\
\hline $\mathrm{C}_{2}$ & 33.00 & 33.88 & 32.44 & 33.12 & 30.12 \\
\hline $\mathrm{C}_{3}$ & 33.55 & 20.11 & 26.22 & 23.29 & 23.29 \\
\hline $\mathrm{C}_{4}$ & 30.88 & 34.66 & 39.55 & 35.03 & 31.07 \\
\hline $\mathrm{C}_{5}$ & 24.55 & 24.11 & 24.55 & 24.40 & 23.09 \\
\hline $\mathrm{C}_{6}$ & 33.33 & 32.88 & 33.33 & 33.18 & 33.58 \\
\hline $\mathrm{C}_{7}$ & 40.40 & 40.00 & 40.77 & 40.39 & 43.77 \\
\hline $\mathrm{C}_{8}$ & 28.44 & 29.33 & 28.88 & 28.88 & 25.77 \\
\hline $\mathrm{C}_{9}$ & 39.11 & 34.67 & 38.22 & 37.33 & 34.39 \\
\hline $\mathrm{C}_{10}$ & 29.33 & 30.11 & 29.22 & 29.55 & 26.16 \\
\hline $\mathrm{C}_{11}$ & 29.11 & 28.66 & 30.44 & 29.40 & 26.58 \\
\hline $\mathrm{C}_{12}$ & 25.00 & 25.66 & 25.88 & 25.51 & 23.98 \\
\hline $\mathrm{C}_{13}$ & 32.00 & 32.44 & 33.77 & 32.77 & 32.85 \\
\hline $\mathrm{C}_{14}$ & 27.11 & 28.11 & 29.11 & 28.11 & 26.80 \\
\hline $\mathrm{C}_{15}$ & 28.00 & 28.88 & 28.88 & 28.59 & 27.82 \\
\hline
\end{tabular}




\section{THE REGRESSION EQUATION}

The solution of equation 19, given the responses in table 3, gives the unknown coefficient of the regression equation as follows:

$$
\begin{array}{lll}
\propto_{1}=-5685.38 & \propto_{12}=3415.259 & \propto_{24}=835.9397 \\
\propto_{2}=-6692.39 & \propto_{13}=-3595.77 & \propto_{25}=553.3372 \\
\propto_{3}=21943.39 & \propto_{14}=-566.255 & \propto_{34}=-1386.23 \\
\propto_{4}=771.4913 & \propto_{15}=814.8985 & \propto_{35}=-2371.04 \\
\propto_{5}=-18.9654 & \propto_{23}=-2049.49 & \propto_{45}=-72.7589
\end{array}
$$

Hence, from equation $15 \mathrm{~b}$, the regression equation is given by:

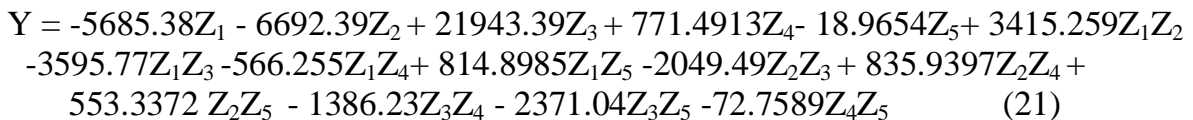

Equation (21) is the mathematical model for the optimization of compressive strength of Pulverised Fuel Ashcement concrete based on Osadebe's second-degree polynomial.

\section{TEST OF ADEQUACY OF THE MODEL}

The test for adequacy for Osadebe's Regression Model was done using Fischer test at 95\% accuracy level. The compressive strength at the control points

(i.e. $\mathrm{C}_{1}, \mathrm{C}_{2}, \mathrm{C}_{3}, \mathrm{C}_{4}, \mathrm{C}_{5}, \mathrm{C}_{6}, \mathrm{C}_{7}, \mathrm{C}_{8}, \mathrm{C}_{9}, \mathrm{C}_{10}, \mathrm{C}_{11}, \mathrm{C}_{12}, \mathrm{C}_{13}, \mathrm{C}_{14}, \mathrm{C}_{15}$ ). In this, two hypotheses were set aside:

a) Null Hypothesis: At $95 \%$ accuracy level, that there is no significant difference between the laboratory concrete cube strength and model predicted strength results.

\begin{tabular}{|c|c|c|c|c|c|c|}
\hline $\begin{array}{l}\text { Control } \\
\text { points }\end{array}$ & $\mathbf{y}_{\mathbf{e}}$ & $\mathbf{y}_{\mathbf{m}}$ & $\mathbf{y}_{e}-\bar{y}_{e}$ & $\mathbf{y}_{\mathbf{m}}-\overline{\mathbf{y}}_{\mathbf{m}}$ & $\left(\mathbf{y}_{\mathrm{e}}-\overline{\mathbf{y}}_{\mathrm{e}}\right)^{2}$ & $\left(\mathbf{y}_{m}-\bar{y}_{m}\right)^{2}$ \\
\hline $\mathrm{C}_{1}$ & 41.44 & 41.38 & 10.13 & 11.34 & 102.62 & 128.69 \\
\hline $\mathrm{C}_{2}$ & 33.11 & 30.12 & 1.8 & 0.08 & 3.24 & 0.006 \\
\hline $\mathrm{C}_{3}$ & 23.29 & 23.39 & -8.02 & -6.63 & 64.32 & 44.22 \\
\hline $\mathrm{C}_{4}$ & 33.69 & 31.07 & 2.38 & 1.03 & 5.66 & 1.06 \\
\hline $\mathrm{C}_{5}$ & 24.40 & 23.09 & -6.91 & -6.95 & 47.75 & 48.30 \\
\hline $\mathrm{C}_{6}$ & 33.14 & 33.38 & 1.86 & 3.34 & 3.46 & 11.16 \\
\hline $\mathrm{C}_{7}$ & 40.39 & 43.77 & 9.08 & 13.73 & 82.45 & 88.51 \\
\hline $\mathrm{C}_{8}$ & 28.88 & 25.77 & -2.43 & -4.27 & 5.90 & 18.23 \\
\hline $\mathrm{C}_{9}$ & 37.33 & 34.39 & 6.02 & 4.35 & 36.24 & 18.92 \\
\hline $\mathrm{C}_{10}$ & 29.55 & 26.16 & -1.39 & -3.88 & 1.93 & 15.05 \\
\hline $\mathrm{C}_{11}$ & 29.40 & 26.58 & -1.91 & -3.46 & 3.65 & 11.97 \\
\hline $\mathrm{C}_{12}$ & 25.51 & 23.98 & -5.8 & -6.06 & 33.64 & 36.72 \\
\hline $\mathrm{C}_{13}$ & 32.73 & 32.85 & 1.43 & 2.81 & 2.04 & 7.80 \\
\hline $\mathrm{C}_{14}$ & 28.11 & 26.80 & -3.20 & -3.29 & 10.24 & 10.50 \\
\hline $\mathrm{C}_{15}$ & 28.59 & 27.82 & -2.72 & -2.22 & 7.40 & 4.93 \\
\hline Sum & $\begin{array}{l}\sum \mathrm{ye} \\
=469.6\end{array}$ & $\begin{array}{l}\sum y m \\
=450.55\end{array}$ & & & $\begin{array}{c}\sum(\text { ye }-\overline{y e})^{2}= \\
410.54\end{array}$ & $\begin{array}{c}\sum(\mathrm{ym}- \\
\left.\overline{\mathrm{y}}_{\mathrm{m}}\right) 2= \\
546.076\end{array}$ \\
\hline Mean & $\overline{\mathrm{y} e}=31.31$ & $\overline{\mathrm{y}}_{\mathrm{m}}=30.04$ & & & & \\
\hline
\end{tabular}

b) Alternative Hypothesis: At $95 \%$ accuracy level, there is a significant difference between the laboratory concrete cube strength and model predicted strength results.

The test is carried out as shown in Table 5 .

Table 5: Fischer-statistical test computations for Osadebe's Regression Model

Note: ye is the experimental compressive strength, while ym is the model compressive strength

$\mathrm{S}_{\mathrm{e}}{ }^{2}=\frac{\sum(\mathrm{ye}-\overline{\mathrm{y} e})^{2}}{\mathrm{~N}-1}=\frac{410.39}{14}=29.32$ 
$\mathrm{S}_{\mathrm{m}}^{2}=\frac{\sum(\mathrm{ym}-\overline{\mathrm{y} m})^{2}}{\mathrm{~N}-1}=\frac{546.076}{14}=39.00$

$\mathrm{F}_{\text {calculated }}=\frac{\mathrm{S}_{1}{ }^{2}}{S_{2}{ }^{2}}$

Where $\mathrm{S}_{1}{ }^{2}$ is the greater of $\mathrm{S}_{\mathrm{e}}{ }^{2}$ and $\mathrm{S}_{\mathrm{m}}{ }^{2}$, while $\mathrm{S}_{2}{ }^{2}$ is the smaller of the two.

Here $\mathrm{S}_{1}{ }^{2}=\mathrm{S}_{\mathrm{m}}{ }^{2}=39.00$ and $\mathrm{S}_{2}{ }^{2}=\mathrm{S}_{\mathrm{e}}{ }^{2}=29.32$

$\mathrm{F}_{\text {calculated }}=\frac{39.00}{29.32}=1.330$

The model is acceptable at $95 \%$ confidence level if:

$\frac{1}{F_{\propto(V 1, V 2)}}<\frac{\mathrm{s}_{1}{ }^{2}}{S_{2}{ }^{2}}<F_{\propto(V 1, V 2)}$

Where

Significant level, $\propto=1-0.95=0.05$

Degree of freedom, $\mathrm{V}=\mathrm{N}-1=15-1=14$

From standard F-statistic table, $F_{\propto(V 1, V 2)}=2.443 \quad$ and.

$\frac{1}{F_{\propto(V 1, V 2)}}=\frac{1}{2.443}=0.4093$

Hence the condition: $\frac{1}{F_{\propto(V 1, V 2)}}<\frac{\mathrm{s}_{1}{ }^{2}}{S_{2}{ }^{2}}<F_{\propto(V 1, V 2)}$ which is $0.4093<1.330<2.443$ is satisfied.

Therefore, the null hypothesis that "there is no significant difference between the experimental and the model expected result" is accepted. This implies that Osadebe's Regression Model is adequate.

\section{Conclusion}

Using Osadebe's second degree polynomial regression equation, mix design model for a five component Pulverised Fuel Ash- cement concrete cube was developed. This model could predict the compressive strength of PFA-Cement concrete cube when the mix ratios are known and vice versa. The predictions from this model were tested at 95\% accuracy level using statistical Fischer test and found to be adequate.

\section{References}

[1]. Afolayan, J.O. and Alhassan , A.Y. (2010) "Durability of Concrete Containing Pulverised Fuel Ash in Aggressive Environment" Journals of Engineering and Applied Science. Vol. 2

[2]. BS 12: (1978), "Specification for Portland cement".

[3]. Neville, A.M., (1996), "Properties of Concrete", Longman Ltd., England

[4]. Neville, A.M., and Brook, J.J.,( 1990), "Concrete Technology”, Longman Ltd., Singapore

[5]. Scheffe, H. (1958), "Experiments with Mixtures", Journal of the Royal Statistical Society. Ser. B., 20, 344 - 360.

[6]. Obam, S.O.(2009). "A mathematical model for Optimization of Strength of Concrete: A case study for shear modulus of Rice Husk Ash Concrete. Journal of Industrial Engineering International. Vol.5 (9) 76-84

[7]. Osadebe, N.N., (2003). "Generalized Mathematical Modelling of Compressive Strength of Normal Concrete as Multi-variate function of the Properties of its Constituents components". A paper delivered at college of Engineering, University of Nigeria Nsukka.

[8]. Uwe, B. (2010). " Pozzolans: Trash to Treasure, http:// ferrocement.net/ferro/pozzolans 